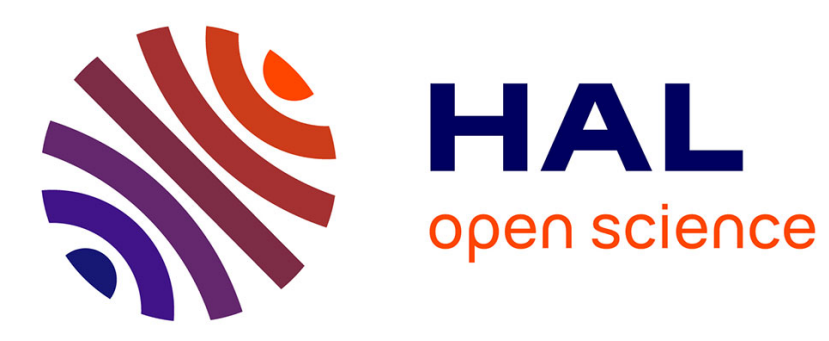

\title{
Le pouvoir obscur de la délation
}

Fabien Jobard, Jean-Paul Brodeur

\section{To cite this version:}

Fabien Jobard, Jean-Paul Brodeur. Le pouvoir obscur de la délation. Jean-Paul Brodeur; Fabien Jobard (dir.). Citoyens et délateurs. La délation peut-elle être civique?, Autrement, pp.195-211, 2005, Mutations, 2-7467-0728-4. 10.3917/autre.brode.2005.01.0195 . halshs-00005072

\section{HAL Id: halshs-00005072 https://shs.hal.science/halshs-00005072}

Submitted on 2 Jul 2015

HAL is a multi-disciplinary open access archive for the deposit and dissemination of scientific research documents, whether they are published or not. The documents may come from teaching and research institutions in France or abroad, or from public or private research centers.
L'archive ouverte pluridisciplinaire HAL, est destinée au dépôt et à la diffusion de documents scientifiques de niveau recherche, publiés ou non, émanant des établissements d'enseignement et de recherche français ou étrangers, des laboratoires publics ou privés. 


\section{Le pouvoir obscur de la délation}

Fabien Jobard

Jean-Paul Brodeur

Embrasser d'un regard les situations les plus diverses et les lieux historiques les plus éloignés que traversent les textes que nous avons présentés dans ce volume offre l'étrange impression de la répétition. S'il fallait trouver un personnage politique qui prît les traits d'un universel, le délateur serait à coup sûr celui-là. Sycophante de la cité athénienne ou delator de la Rome républicaine, un visage a vu le jour qui n'eut de cesse, sous les ors des palais ou au sein du peuple tout entier, de renaître chaque fois presque inchangé, intact. Ce ne sont pas seulement les traits funestes du délateur qui reviennent dans les tourments de l'histoire, mais bien une technique épurée de pouvoir. Ce que montrent en effet les textes parcourus dans ce volume, c'est autant la constance du dégoût suscité par le délateur, que la répétition selon les régimes politiques de la délation comme technique politique.

Répétition, ainsi, de ce mouvement qui veut que la délation est honnie sitôt qu'elle est promue, et par ceux-là même qui l'ont promue: Yann Rivière rappelle que l'on adopta une disposition, sous Constantin, qui promettait la mort au délateur, "homme de lucre et de sang », s'il venait seulement à trop dénoncer. François-Xavier Nérardévoque également ce perpétuel balancier du pouvoir : Pierre le Grand qui adopte en 1713 un oukase incitant à la dénonciation prend sans attendre un second oukase, en 1715, qui condamne la dénonciation... sauf pour les affaires «importantes ${ }^{1}$.

Répétition, aussi, du grotesque de la délation, prise dans une économie impossible, en tenaille entre l'insatiabilité et la circularité. Insatiabilité de cette pratique : qui emploie le délateur, semble toujours craindre plus ce qu'il ignore encore à mesure qu'il apprend davantage. La délation qui est l'arme des tyrans est aussi toujours leur passion. Conséquence : la circularité de la délation qui, de fin au service du pouvoir, devient un moyen de se protéger de ce qu'elle génère. On voit ainsi le Prince inquiet du délateur tenter de circonscrire son pouvoir néfaste en organisant une bureaucratie de la plainte et de la dénonciation au sein de laquelle chacun en vient à surveiller tout le monde, dans un système pyramidal qui à force de vouloir tout épier ne voit plus rien. La Stasi de la RDA, que décrit Sonia Combe, semble définitivement la perfection formelle d'un système que la perfection, justement, étouffa.

La RDA fut comme une sorte de terre élective de ces théâtres grotesques, de ces anecdotes qui soulignent l'impuissance d'un régime fondé sur la surveillance mutuelle et permanente. Il faut ainsi relire l'anecdote rapportée par Sonia Combe dans son article, où trois agents délateurs, au cours d'une soirée festive que le pouvoir soupçonne d'abriter quelque poussée conspirative, s'épient mutuellement, chacun intrigué par le comportement suspect du délateur qu'il observe. Ce n'est pas ici le pouvoir qui est en cause, mais la pratique elle-même de la délation qui, à quelque échelle qu'on la prenne, est toujours susceptible de succomber au grotesque, fruit de son caractère circulaire.

Le ridicule ne tue pas la délation, ni malheureusement son caractère odieux : tout ceci ne doit pas faire oublier le caractère intéressé, vénal, en un mot méprisable de la délation, qui signesa distinction par rapport au terme «dénonciation ». C'est bien à l'aune de cela qu'il faut juger

\footnotetext{
${ }^{1}$ François-Xavier Nérard, $5 \%$ de vérité. La dénonciation dans l'URSS de Staline. Paris : Tallandier, 2004, chap. 1.
} 
l'encouragement de la délation par le pouvoir. Pour autant, associer systématiquement la délation au pouvoir ne permet pas de faire le partage entre toutes les formes de dénonciation qui ont été présentées ici, et qui ont pour autant toute leur place sous l'appellation de délation. Le "outing» pratiqué en Amérique, puis en Europe, ne s'est-il pas révélé comme l'arme ultime employées par des victimes que l'on prive de l'arme du "visage découvert», comme le souligne la tribune d'Act Up Paris que nous avons reproduite ? Lorsque l'enjeu, en effet, est de ne pas pouvoir se dire tel que l'on se vit (en l'occurrence, homosexuel), la dénonciation ne devient-elle pas le seul moyen de lutter, précisément, à armes égales contre ceux qui, de toutes façons, voient leur vie privée protégée, sanctuarisée, consacrée tant par la loi que par les usages? Le texte de Anne-Lise Ulmann sur ces lettres de délation qui parviennent aux Caisses d'allocation familiale ne montre-t-il pas, également, l'exiguïté des formes disponibles, des manières convenables de protester lorsque l'on a peu ? Ne montre-t-il pas également, dans l'embarras de la CNAF à codifier les réponses convenables aux lettres de dénonciation, la difficulté à trancher avec certitude entre les statuts de victime et de juge ?

C'est à ce point de la réflexion qu'il faut un peu d'ordre. La délation est d'abord une arme de l'Etat, ou du pouvoir; mais en son sein-même une arme bifide, pouvant être tout aussi bien employée par le Prince au sein même de l'Etat contre, précisément, ses propres agents. Nous insisterons ensuite sur les nouvelles modalités de la délation, et les nouveaux rapports, alors, de la délation, du pouvoir et de la morale. Nous verrons ainsi dans quelle mesure la délation apparaît aujourd'hui comme une arme au service de la société civile, en précisant bien sûrles limites de cette arme toujours à double tranchant, quelle que soit la main qui la manipule.

\section{1) La délation à la genèse de l'Etat}

Qu'est-ce qu'un Etat? C'est l'organisation qui revendique, selon la formule de Max Weber, le «monopole de la violence physique ». C'est ainsi l'organisation qui assure le monopole de la sanction (seul l'Etat peut tuer, avec l'individu en cas exceptionnel de légitime défense). Une telle mise au point serait parfaitement futile, si le texte de Yann Rivière ne nous rappelait cette évidence: l'Etat est aussi l'institution qui revendique le monopole de la menace de sanction. Et c'est là que la délation participe au succès, ou à l'échec, de la construction de l'Etat. Par menace de la sanction, il faut entendre ici les chances d'être poursuivi si l'on a commis une faute. Or, à leur naissance, les Etats ne disposaient pas de force nécessaire à l'établissement d'une autorité capable de poursuivre avec succès les individus fautifs, ou présumés tels. On était tributaire, alors, du citoyen individuel, à qui l'on demandait de rapporter, de dénoncer. Cette compétence de fait du citoyen, cette sorte (pour employer et dévoyer un terme juridique contemporain) de délégation de pouvoir judiciaire, s'étendait également à l'autre "nerf » de l'Etat: sa capacité à prélever ses ressources par l'impôt. Là encore, le délateur était requis pour signaler ceux qui esquivaient l'impôt obligatoire.

Ce n'est qu'une fois l'Etat constitué, qu'une fois l'accumulation de ses ressources assurée, qu'il put former une autorité autonome en charge de la poursuite et de l'enquête, fiscales ou judiciaires. Les «fiskaly»², sous Pierre le Grand, ont précédé de deux ans l'oukase créant l'obligation de délation que nous avons évoqué. Prélèvement obligatoire, pouvoir d'exercer la violence physique ${ }^{3}$ : les deux piliers de l'Etat voient leur naissance intimement liée à la figure du délateur. L'administration fiscale ou l'autorité judiciaire voient ainsi dans le délateur un peu de leur origine constitutive. Est-ce cette genèse déjà honteuse, puisque Yann Rivière nous

\footnotetext{
${ }^{2}$ Puisant à la même origine que le français (le «fiscus » latin était un panier destiné à recevoir de l'argent), le terme désigne le Trésor de l'Etat (cf. Nérard, op. cit., p. 28).

${ }^{3}$ Sur la genèse de l'Etat et la captation des ressources, voir Charles Tilly in Politix.
} 
montre à quel point les délateurs étaient loués autant qu'abhorrés, qui fait aujourd'hui encore l'embarras de l'Etat face à la délation ?

\section{2) De la délation à l'administration (aller-retour)}

a) Contre les langues bien pendues : l'œil de l'Etat

La délation est insatiable. Que faire pour qu'elle n'en vienne à ruiner les fondements de la puissance publique? L'Etat institutionnalise alors ses mécanismes de fonctionnement, se protège des individus toujours suspects de vénalité ou de mauvaises intentions,et créé une administration, une bureaucratie. Dans un ouvrage remarquable, Paolo Napoli montre ainsi que ce qui caractérise l'Etat moderne, par exemple l'Etat français, c'est bien la police. Non pas la police entendue, justement, comme l'institution des mouchards et des espions (cette police-là aura sa belle naissance, mais plus tard), mais la police entendue comme l'administration en charge de la surveillance de la société : surveillance du travail (des marchés, des corporations), surveillance des idées (la censure), surveillance des échanges (des prix) ${ }^{4}$. C'est la police (qui contrôle) qui donne naissance à l'administration (qui régule) : la censure, par exemple, est devenue simple obligation de déclaration de publication (ainsi le $\mathrm{n}^{\circ}$ ISBN qui figure en quatrième de couverture de cet ouvrage, dont trois exemplaires ont été envoyés à la préfecture). Les figures du veilleur embusqué ou du traître rémunéré font place à celles, plus bonhommes, des bureaucrates qui enregistrent, classent, estampillent, houspillent.

L'administration, c'est l'œil de l'Etat... Plutôt que déléguer à des délateurs ou des sycophantes, des indics ou des tontons, l'Etat préfère la routine administrative. Cette routine lui permetde mieux connaître sa société. Administrer les choses, voilà qui est plus efficace qu'interroger les gens : si l'on censure, on ne sait pas la confiance qu'accorde le peuple au journal qu'on interdit de publication. Mais si on surveille sa parution et sa diffusion, on saura mieux quel écho le journal emporte dans une société qu'on veut connaître pour mieux la maîtriser... Laissons paraître, cessons d'épier : "la liberté de la presse servira à la fois les souverains et les peuples. A ceux-ci elle sera une garantie, à ceux-là un moyen de gouvernement », disait Guizot, ministre libéral favorable au début du XIXe siècle à l'ouverture de la monarchie constitutionnelle française.

\section{b) Faut-il faire confiance aux agents de l'Etat?}

Le pouvoir exorbitant du délateur est évincé par la mise en place de la puissance administrative. Mais la question de la loyauté ne fait que se déplacer : et si l'administration usait mal de son pouvoir? Il faut alors contourner cette nouvelle puissance et en percer les intentions. L'affaire n'est pas simple car c'est tout le service administratif que la faute individuelle met en péril ; non pas que tous soient complices, mais le supérieur hiérarchique, s'il n'a découvert la faute de son subordonné, est ainsi coupable de défaut de vigilance... On ne peut se reposer sur l'administration pour contrôler l'administration. Comment faire?

Les dictatures ont tranché : construire une pluralité d'appareils administratifs chargés de se neutraliser l'un l'autre: l'Etat hitlérien incarne une sorte de modèle de duplication systématique des autorités. Mais les démocraties? Prenons le problème épineux de la surveillance des policiers. La France est particulièrement exposée sur ce point, qui est l'un des deux pays à jamais s'être fait condamner pour «torture » de la part de policiers par la Cour européenne des droits de l'homme, en juillet 1999. La Cour pointait non seulement la gravité

\footnotetext{
${ }^{4}$ Paolo Napoli, Naissance de la police moderne. Pouvoir, normes, société. Paris : La Découverte, 2003.
} 
des exactions policières, mais aussi l'insigne lenteur des autorités administratives (la police des polices) et judiciaires à instruire sur les fautes commises par des agents de l'Etat.

Désireux de donner des gages de police démocratique, ouverte au regard de tous, Lionel Jospin annonça lors de son discours d'investiture en 1997 la création d'une haute autorité de contrôle, indépendante. Trois ans après, la loi fut votée (le 10 juin 2000) et la Commission nationale de déontologie de la sécurité fut mise en place 5 . Elle entend des policiers (mais aussi des gendarmes, des gardiens de prison, des personnels privés) dans les affaires de manquement grave à la déontologie. Danger! Et si les agents mettaient leur hiérarchie à l'index, le fonctionnement de leur service, l'impéritie de leurs supérieurs? Inquiète, la direction centrale de la police émit récemment une note de service précisant que même si un agent est libre de se faire accompagner de la personne de son choix à la CNDS, il était fermement encouragé qu'il soit secondé par un «membre de sa hiérarchie» ${ }^{6} . .$. Et voilà l'institution qui se protège des regards extérieurs, en muselant la voix de ses agents. En Amérique du Nord, où les commissions d'enquête sont légion pour éviter les omertas d'Etat, les témoins, informateurs, indicateurs, des commissions sont fermement protégés par la loi. Comment travailleraient en effet ces instances s'elles ne pouvaient s'assurer de la loyauté d'informateurs infiltrés, ou ne pouvaient garantir la protection d'agents retournés ?

Parce que l'administration est elle aussi une organisation faite d'opacités insondables, parce qu'elle abrite également des services dont les actions sont particulièrement sensibles (services de police, notamment), les armes choisies pour y traquer les erreurs ou les dérives sont les mêmes que celles en matière de lutte contre la délinquance organisée ou le crime en col blanc : l'infiltration, le témoignage protégé ou anonyme, etc. En matière de lutte contre les errements de ses propres agents, l'Etat encourage aujourd'hui d'une main ce qu'il défend d'une autre: l'exemple de la CNDS en est un, mais on pourrait en citer bien d'autres'.

\section{3) L'Etat, ou le pouvoir?}

Voilà une histoire bien lisse : la puissance publique, en situation d'impuissance budgétaire, ne peut se reposer que sur la délation; mettant ainsi la société tout entière en danger, elle met sur pied l'administration qui fait écran entre elle et les citoyens tentés par le vice de la délation. Puis la puissance publique retrouve la nécessité de la délation, qu'elle encourage et freine dans le même mouvement. Mais cette histoire ne distingue pas l'Etat du titulaire du pouvoir, le prince ou le souverain. Dans les Etats qui n'offrent pas, par l'élection, ce contrôle minimal du souverain par les gouvernés, le principe du gouvernement est l'omnipotente méfiance du prince, qui veille sans cesse à ce que personne ne le menace. On retrouve, là encore, les oukases de l'empereur de toutes les Russies, l'usage massif de la délation par la dictature nazie ou l'encouragement à la délation, de manière générale, lorsque se met en place un nouveau régime, lorsque les règles de distribution des pouvoirs sont mal définies, laissant toutes les craintes s'emparer des titulaires encore incertains des places et des postes.

\footnotetext{
${ }^{5}$ Ses rapports sont toujours instructifs : on peut les consulter sur www.cnds.fr.

${ }^{6}$ Direction centrale de la sécurité publique, Note PN/CAB/n ${ }^{\circ} 04-8107$ du 5 juillet 2004.

${ }^{7}$ Ainsi du devoir de désobéissance des fonctionnaires, lorsqu'on les oblige à exécuter un ordre contraire aux principes fondamentaux. La loi encourage la désobéissance (loi portant statut des fonctionnaires, du 13 juillet 1983) ; mais le Conseil d'Etat rappelle avec constance qu'en la matière la défense de « l'intérêt du service » est l'élément décisif autorisant la désobéissance, et non pas l'ordre illégal : c'est la puissance publique que l'on protège, plus que les administrés (cf. Eric Desmons, Droit et devoir de résistance en droit interne. Paris : LGDJ, 1999 , p. 166-178).
} 
Ce n'est pas seulement la Terreur qui, sous la Révolution française, libéra les forces incontrôlées de la délation, mais le changement de régime. Camille Desmoulins voulait "détruire ce préjugé qu'il y a de la honte à être délateur ». Marat, dans son journal L'Ami du peuple, encourageait la délation contre « le premier ministre jusqu'au dernier commis » comme un «droit naturel ». Mais c'est dès 1789, lorsque le pouvoir est incertain, lorsque le pouvoir se cherche, se craint,que la délation occupe une bonne place des débats de l'Assemblée nationale. "Il faut aujourd'hui établir entre [la police et les citoyens] le plus de rapports possibles ; il faut établir la communauté des citoyens », explique le rapporteur Duport, député de Paris, à la tribune du projet de loi sur la police, fin $1789^{8}$. On en appelle à la communauté civique, réchauffée par les voix innocentes des dénonciateurs et délateurs.

L'Etat n'est pas ici en jeu, mais le nouveau titulaire du pouvoir, qui peu sûr de sa pérennité cherche ses alliés du regard, épie, surveille, redécouvre les pratiques de cour et de secret : l'art d'observer ses semblables, de simuler et de dissimuler, de dire et de médire, de se permettre de rapporter un petit fait ou de laisser éclater un grand scandale, à la mesure de la position que l'on occupe et de la position que l'on vise. Le Roi croira-t-il ce qu'on lui a dit de son ministre, ou de son chambellan? Continuera-t-il à accorder à ce dernier le privilège de lui ôter sa chemise au moment du coucher ? ${ }^{9}$ S'il croit Untel sur parole, n'est-ce pas qu'il lui confère du pouvoir ou, ce qui est la même chose alors, du crédit, de l'honneur ? Le président de la République gardera-t-il son ministre dont on vient de faire savoir qu'il loge dans un appartement de fonction de plus de $600 \mathrm{~m}^{2}$ ? Plus important encore (car des ministres, il s'en trouve toujours) : gardera- $t-i l$ sa confiance au ministre de l'Economie et des Finances des services duquel filtra la révélation fatale? Qui se tapit encore au ministère dont il faudrait se défaire? Quels visages épier, quels mécanismes employer ? ${ }^{10}$

Quelles que soient les procédures administratives que l'Etat met en place pour se protéger et protéger ses administrés de l'infamie, l'exercice du pouvoir garde une irréductible part de secret, ménageanttoujours une place, dans l'arsenal des tactiques politiques, au délateur. Délateur que l'on retrouve encorelorsque l'Etat veut décrypter sa propre administration, ou lorsque le gouvernant veut connaître le peuple, au-delà des rapports que lui adresse son administration. C'est toute la logique russe, puis soviétique, de la procédure de «signal », qui permet au souverain de se protéger d'une administration pléthorique ou menaçante, pour assurer également un lien simulacre de lien «immédiat » avec le peuple dans un système sans élection - un lien de subordination démagogique, dans lequel chacun est appelé à dénoncer le puissant local, l'administrateur corrompu, le bureaucrate aveugle.

Il y a un stratagème toujours tentant dans l'appel à la délation contre les titulaires de charges que l'on a soi-même distribuées, dont l'expression la plus spectaculaire et parmi les plus meurtrières fut la Révolution culturelle chinoise ${ }^{11}$. Forme suprême du gouvernement par la démagogie, l'appel à la délation contre sa propre administration ressuscite la figure du délateur jouissant sous l'oripeau civiquede sa propre vénalité. La démocratie rend plus complexe la question de la délation. En dictature, l'affaire est simple : la délation est une arme du prince contre ceux qui conspirent, elle est aussi l'arme pour mieux contrôlerson administration et ses subordonnés, par un simulacre d'appel à ceux «d'en-bas», au «petit peuple». Mais en

\footnotetext{
${ }^{8}$ Cité in Napoli, op. cit., p. 208.

${ }^{9}$ Sur le fonctionnement de la société de Cour et les mécanismes de distribution des chances de puissance, voir Norbert Elias, La société de cour. Paris : Flammarion-Champs, 1985 (traduit de l'allemand, 1969), p. 70-72.

${ }^{10}$ Ou bien : « qui donc a porté à la connaissance de la presse la funeste arrestation du collaborateur du Premier ministre surpris en curieuse compagnie sur les boulevards ?»...

${ }^{11}$ Rappelons toutefois que la délation reste une vertu du régime, puisqu'une « Journée de la délation » reste célébrée, une fois par an.
} 
démocratie ? Là existent la presse, l'élection, l'opinion publique... En somme, un espace public est établi qui permet à l'opinion juste de se former. L'espace public est ce lieu idéal de formation civique de l'opinion et de la décision. Pourquoi subsistent alors des pratiques de délation en démocratie, lorsque tout devrait y assurer la circulation de la parole ?

\section{4) La délation, l'arme des sans voix ?}

Luc Boltanski dit de l'espace publique qu'il «suppose la constitution d'un observateur détaché, dégagé, sans engagement préalable et surtout sans attaches locales, capable de surplomber les singularités de la société » ${ }^{12}$. L'espace public s'incarne dans des impossibles sociaux: qui bénéficie de l'aérien détachement qui permet la dénonciation informée, juste et surtout dépersonnalisée d'une injustice, au profit d'un but qui dépasse l'intérêt circonscrit de celui qui dénonce; une dénonciation désintéressée d'une cause juste et universelle ? Et sachant que l'acte de dénoncer coûte (il oblige à s'engager, à interpeller, à faire savoir, à mobiliser), qui aura la force, les ressources ou le désintérêt suffisants pour dénoncer sans faire œuvre de délateur?

Carle plus court chemin de la dénonciation d'une inéquité ou d'un préjudice, c'est bien la délation à l'encontre de celui que l'on identifie, à tort ou à raison, comme l'auteur du préjudice. A nouveau, on dénonce le puissant ou le voisin. Par delà la nature du régime politique dans lequel on vit, toujours le même mécanisme: à l'appel au signalement des dysfonctionnements administratifs répondent les dénonciations du voisin, au nom de sa supériorité en ceci ou cela. "La fuite dans le général laisse insatisfaite l'incitation qui pousse à dénoncer le ou les persécuteurs responsables de l'injustice et à faire savoir l'intensité des souffrances endurées », dit encore Boltanski. D'ailleurs, ne soupçonne-t-on pas le riche qui dénonce la pauvreté, et plus généralement tous ceux qui «parlent sans savoir » ou "parlent sans souffrir » de ne le faire que par intérêt à se faire connaître, ou de capter à leur propre cause la parole de ceux qui sont reclus au silence ? La délation, l'arme des « sans »?

Ce que la délation recouvre alors, c'est le caractère non accompli des conditions d'exercice de la liberté; par exemple les conditions de jouissance de la liberté d'être soi. C'est ce que rappelle avec force le texte d'Act Up Paris où l'organisation retourne l'accusation de délation qu'on lui impute, en pointant précisément les conditions préalables de prise de parole sur soi, les conditions inégales de l'accomplissement de soi, dans la société d'aujourd'hui, sous l'apparence de l'espace public. Les conditions d'exercice de la liberté, ce sont aussi les conditions d'accès au savoir, les conditions d'accès aux ressources qui permettent d'être détaché des contingences et de formuler la dénonciation de l'injustice dans les cadres requis pour être entendue.

L'embarras de la Caisse nationale d'allocations familiales à formuler une ligne de conduite face aux lettres de délation qu'on lui adresse s'inscrit dans la continuité de la difficulté de la puissance publique à se faire autonome à l'égard des citoyens (d'où l'insistance que formule la CNAF, dans la circulaire que reproduit Anne-Lise Ulmann, sur «la crédibilité de l'institution »). Mais il témoigne aussi de la répétition de l'impossible exigence : la délation est toujours justifiée lorsque à la fois les faits sont « graves » et les motivations pures ( à côté de signalements de faits sans gravité, inspirés par la jalousie, la vengeance etc... coexistent des dénonciations de faits graves, de délits portant sur l'attribution de prestations légales »). Mais comment poser pour toujours les règles de correspondance entre la pureté des intentions et la

\footnotetext{
${ }^{12}$ Luc Boltanski, La souffrance à distance. Paris : Métaillé, p. 51.
} 
taille de l'atteinte? N'est-ce pas encore exiger l'impossible détachement, appeler le lésé à la vertu civique, l'apanage de ceux qui ne souffrent pas?

\section{5) Les visages neufs de la délation}

Ces questions constituent aujourd'hui un enjeu considérable car, les textes présentés l'ont montré, la délation se déploie aujourd'hui sous des apparences nouvelles, et produisent parfois des effets de masse, jusque dans les démocraties contemporaines. Il y plus de 2 millions de personnes incarcérées aux Etats-Unis, et les infractions en matière de stupéfiants constituent la source la plus grande d'approvisionnement de l'incarcération aux USA. Or, comme on l'a vu dans le chapitre de Peter Manning, la plus grande partie de l'élucidation des affaires de stupéfiants découle de la collaboration de délateurs. Aujourd'hui, un répertoire renouvelé se met en place, qui laisse apparaître les éléments suivants :

a) Le savoir. En dépit du secret qui protège l'anonymat des délateurs, nous connaissons beaucoup mieux la délation. Peter Manning et son équipe ont pu conduire des recherches dans les archives de plusieurs grands services de police des Etats-Unis. Il en va de même pour l'un d'entre nous (JPB), qui a également dépouillé les archives où étaient déposées les informations fournis par des indicateurs de haute police de trois grandes forces policières canadiennes. Toutes ces recherches ont fait ressortir la distinction entre plusieurs types de dénonciations, en particulier la distinction fondamentale entre l'indicateur et le délateur (dans son sens précis de témoin "spécial et protégé ; voir les entretiens avec PinoArlacchi et Denis Boivin).

b) Le destinataire. Tout lieu où s'échange du pouvoir abrite des velléités de délation. L'Etat, longtemps figure centrale de ce qui se joue autour de la délation, n'est ni le lieu unique, ni le destinataireuniquede ces pratiques. Elles se produisent au sein de l'entreprise privée, de l'École et de l'Université, et de plus en plus souvent au profit de l'opinion publique et de la presse (les lanceurs d'alerte s'adressent fréquemment de façon indifférenciée à l'opinion publique tant nationale qu'internationale). Pour ce qui est de l'Université, on mentionnera que les dénonciations d'enseignants pour « racisme " (ou manque de rectitude politique) ont brisé de nombreuses carrières en Amérique du Nord (le grand roman de Philip Roth, La tache, le rappelle sans détour).

c) La formalisation. La formalisation des relations entre les délateurs et leurs sponsors par le moyen de contrats écrits (qui n'est pas neuve en soi - cf. la République romaine) donne accès tant aux délateurs qu'à leurs employeurs le droit, tout à fait inédit, de recours devant les tribunaux $^{13}$. Cette formalisation est maintenant institutionnalisée en Amérique du Nord. En France, les évolutions sont plus timides: signera-t-on, ne signera-t-on pas, le décret d'application de cet article de la loi Perben II sur la rémunération des délateurs ${ }^{14}$ ?

\footnotetext{
${ }^{13}$ Ce droit est toutefois plutôt exercé par les délateurs que par leurs employeurs, ces derniers disposant de moyens plus expéditifs de faire respecter les contrats. La représentation des délateurs en association professionnelle (cf. entretien avec D. Boivin) est un autre trait de la juridicisation de la délation.

${ }^{14}$ Sachant que par ailleurs, on a confié aux policiers les petites enveloppes permettant la rémunération des balances ; l'essentiel pour la puissance publique, ici comme ailleurs, étant bien d'encourager sans faire savoir. Ou, comme le dit un commentaire de droit : «L'informateur semble marqué au coin d'un paradoxe : alors qu'il est très présent dans la pratique policière, il se trouve dans une zone de non-droit, aucun texte ne venant réglementer le recours à ces individus. Le législateur [=la loi Perben II] se borne à prévoir des questions de rémunération de ces personnes » (Coralie Fiori-Khayat, commentaire de la décision du 9 juillet 2003 de la Cour de cassation, La Gazette du Palais, 17-18 novembre 2004, p. 17).
} 
d) L'organisation. La délation n'est plus seulement affaire d'individus mais elle est devenue une pratique de groupe rationalisée. Ces groupes - comme Act Up ou divers autres d'organismes militant pour le respect de droits spécifiques - obéissent parfois à des motifs tout à fait légitimes, comme le montre Michèle Fournier. Ils opèrent toutefois souvent à partir d'une position d'initié, ce qui est l'une des caractéristiques fondamentales de la délation (voir le chapitre de Jean-Paul Brodeur). Au Canada, où les citoyens possèdent le droit d'obtenir des services dans les deux langues officielles du pays, il se trouve des organismes qui dénoncent systématiquement les manquements à ce droit. Des groupes de délateurs s'organisent pour lutter contre la prolifération de la pornographie infantile. En Pologne, on vient de publier les noms de 162000 personnes liées, ou supposées liées, à l'ancienne police politique du régime communiste, pour lutter contre l'oubli ou pour démasquer les reconvertis trop vite blanchis.

e) L'internationalisation. Ce dernier phénomène est le plus récent. Ainsi de la pratique du outing, pratiquée par l'association Act Up, d'abord fondée aux Etats-Unis. Le cas le plus gros de conséquences de cette migration de la délation réside dans la multiplication des succursales des entreprises multinationales dans divers pays. Toutes ces succursales doivent appliquer les politiques dictées par le siège social, comme par exemple dénoncer les manquements à l'énoncé officiel des valeurs de l'entreprise. Cette politique a été édictée aux États-Unis, après les scandales qui ont mis de grandes entreprises en faillite.

f)Une pénologie clandestine. La plupart des délateurs décrits par Peter Manning purgent une peine substitutive de délation qui leur a été infligée sans procès par les policiers. Ils doivent faire leur choix entre une peine légale d'incarcération imposée par un tribunal ou une peine de délation - également calculée en nombre de mois ou d'années - à laquelle ils sont soumis par la police. Il est à cet égard intéressant de constater que dans le texte anglais original de Peter Manning, le vocabulaire utilisé par les indicateurs pour décrire leur peine de délation est le même que celui des prisonniers pour décrire leur peine d'incarcération.

Tous ces éléments entremêlent et complexifient encore les relations entre pouvoir et délation, pouvoir et société. Essayons de trancher la question, au vu de ces éléments déposés par l'histoire et aujourd'hui transformés, de la délation et du civisme.

\section{6) La délation peut-elle être civique?}

Prenons acte de l'impossibilité sociologique de l'espace public. Que faire ? Libérer les forces de la délation multiplie celles tout aussi néfastes de la démagogie et de la peur. Les effets de la délation se produisent toujours à deux niveaux. S'ils sont dévastateurs pour les individus dénoncés,ils peuvent se révéler bénéfiques pour le reste de la société quand les individus dénoncés sont des prédateurs. Quand les individus dénoncés ne sont que déviants par rapport à des normes arbitraires - par exemple, les préférences sexuelles entre adultes consentants - la délation reste corrosive.

A Athènes, la loi de Solon institua le «ministère public citoyen ». Très vite, certains «accusateurs publics» firent de leur vertu une profession: ces «sycophantes » étaient soit honnis comme contraires à l'esprit de la loi, soit loués comme l'incarnation d'un idéal démocratique achevé. La loi de Solon avait en effet ceci de particulier qu'elle n'obligeait pas à dénoncer lorsqu'une loi de la cité était transgressée (c'est-à-dire lorsqu'une infraction était commise contre l'Etat); elle incitait seulement à prendre la défense d'un concitoyen que l'on estimait lésé dans ses droits. Elle prévoyait ainsi le contournement de la délation pour la satisfaction d'un intérêt personnel en faisant de la délation, d'abord, la défense de la fraternité 
solidaire, la philia, expression d'une démocratie achevée ${ }^{15}$. La délation était, dans ce cas bien particulier, l'instrument de la réhabilitation du lien social, par-delà les envies individuelles, par-delà les communautés particulières.

Le dictionnaire pose la délation comme étant «inspirée par des motifs méprisables ». Or personne ne peut se faire juge des motivations individuelles. Mais pour tracer la ligne qui sépare la délation comme acte indigne et la délation comme acte civique, il faut toujours prendre le geste du délateur dans sa signification d'ensemble; en somme comme un tout, compris tant du côté de l'émetteur que du récepteur. L'acte de dénonciation se distinguerait de celui de délation en ce qu'il ne serait pas pris pour lui-même par le pouvoir, mais seulement pour une injonction à écouter, à retisser le lien social défait par une souffrance individuelle ou collective. En attendant, il est donc impératif de mettre en balance l'acte individuelleet une éventuelle politique de l'incitation. On ne saurait donc souffler à l'oreille des citoyens «caftez sans états d'âme, l'effet compensera l'intention» ou «dites au pouvoir ce que vous savez de votre voisin, le pouvoir rachètera votre souffrance », sans pourrir le civisme.

Mais si la CNAF révisait, après l'étude concertée des lettres de délation, les conditions mêmes de la redistribution des allocations ? Et si la Fédération italienne de football ${ }^{16}$, ou l'Union cycliste internationale, plutôt que jeter ses «traîtres » en pâture à leurs proches ou l'opinion, prenait appui sur les délations (qui sont tant des gestes de rancœur à l'égard de l'adversaire vainqueur-parce-que-dopé que des gestes de désespoir à l'égard de sa propre vie) pour penser au fond la performance, l'humain et le dopage ? Et si le pouvoir polonais considérait la récente publication sur le web d'une liste de 162000 noms de personnes liées, ou supposées liées, à l'ancienne police politique du régime communiste, pour engager un débat de fond sur la mémoire nationale, plutôt que s'en tenir à la simple et bien timorée obligation faite par la loi aux hauts responsables de se dénoncer eux-mêmes. Et si, au fond, les titulaires du pouvoir politique forgeait les instruments permettant l'écoute de ces mille langages politiques inavouables, parce que "mal cadrés», "mal informés», "mal pensant», qui disent sur leur mode propre leur entendement et leur désir politiques ${ }^{17}$ ? De ce point de vue, les manifestations répétées des travailleurs sociaux en 2004 contre le projet de loi de "prévention de la délinquance » visant «l'action concertée » (pudique formulation, encore...) des éducateurs spécialisés et des services de police et de gendarmerie ${ }^{18}$, ou bien l'indignation suscité par l'initiative "Citoyens relais, pourquoi pas vous ? » d'un commissariat de la ville de Douai $^{19}$, disent aussi le désir d'une participation à la vie de la cité qui ne se réduise pas à un rapport de subornation, ici manifestée par «l'obligation de rapporter», des gouvernés aux gouvernants.

Et pour suivre Frédéric Ocqueteau, ne faut-il pas faire état de tendances caractéristiques d'une société en réseau, où la dissémination de l'information est une tendance cardinale? On

\footnotetext{
${ }^{15}$ Carine Doganis, Démocratie et transparence. Les sycophantes et la délation dans la cité d'Athènes à l'époque classique. Paris : Thèse de doctorat de l'Institut d'études politiques, 2004.

${ }^{16}$ Rappelons ainsi le silence de plomb des footballeurs italiens, ainsi que de leur entourage, et la mort précoce, par inexplicables atrophie musculaire ou leucémie, des anciens professionnels Gianluca Signorini (AS Rome), Guido Vincenzi (Sampdoria), Giorgio Rognoni (Milan AC), Bruno Beatrice (Fiorentina)... « Sur 400 joueurs décédés depuis le début des années 1960, près de 70 décès sont suspects » (étude épidémiologique publiée par $L e$ Monde, 16 janvier 2003, p. 23).

${ }^{17}$ Voir de ce point de vue l'ambition de « sociologie du bruit politique » proposée par Philippe Aldrin, Sociologie politique de la rumeur. Paris : PUF, coll. « Sociologie d'aujourd'hui », 2005.

${ }^{18}$ Voir le site du collectif anti-délation : www.abri.org/antidelation.

19 «Le commissaire divisionnaire compte sur des 'citoyens relais' pour seconder la police », Le Monde, 25 novembre 2003, p. 12.
} 
peut considérer l'information sous deux angles différents et complémentaires : le contenu du message communiqué, ou bien le médium lui-même (le téléphone, Internet et ainsi de suite). L’aphorisme de McLuhan selon lequel le médium est le message s'applique dans le domaine de la délation. Les pratiques délatrices ont évolué de façon substantielle avec l'invention de la poste. L'Internet favorise aujourd'hui les langages de la dénonciation (par exemple, les blogs) et de l'intimité (le chat). Une étude conduite par la Commission étasunienne de surveillance des transactions boursières a révélé une croissance exponentielle des dénonciations par Internet et les risques de délation sont décuplés par les capacités des hackers de pénétrer par effraction au sein de systèmes d'information confidentielle. L'innovation ne se résout pas ici à la technique. Elle tient à la motivation des utilisateurs de l'Internet qui dans de très nombreux cas est d'une nature ludique ou relativement gratuite (l'exploit pour l'exploit de casser la sécurité d'un système d'information et de la rendre publique). Une composante essentielle de la motivation des internautes à communiquer entre eux et avec l'univers est au delà du bien et du mal: il s'agit du désir de s'exprimer. En considérant cette possibilité ouverte que la délation constitue un mode d'expression de soi, on pénètre dans une nouvelle sphère dont le risque est qu'elle soit de cristal et ne nous conduise à la transparence totale.

Prise comme le simple indicateur d'une impossibilité d'exprimer une injustice autrement que par la mise à l'index d'un proche ou d'un puissant, la délation devient le signe d'une désaffiliation sociale et politique qui appelle une vigilance redoublée à l'égard de la perte de confiance réciproque des représentants et des gouvernés. L'appel à la délation prend dans nos démocraties médiatisées la forme plus subtile et diffuse d'exigence continue de transparence absolue, et l'Etat qui était l'acteur central de cette incitation cède la place aux opérateurs privés. Or, puisque la délation a toujours été une technique de gouvernement, l'enjeu est aujourd'hui de la déjouer en tant que telle, de mettre au jour ce qui en elle relève volonté de faire de sa propre situation, de sa souffrance, de sa singularité, l'arme d'interpellation publique de la collectivité. C'est là que se joue la responsabilité civique, tant de l'Etat que des canaux privés d'organisation des expressions individuelles et collectives, permettant contre l'appel à la délation ou les injonctions à la transparence de défaire les forces de la dépossession. 